\title{
Biomedical applications of terahertz self-mixing interferometry
}

\begin{abstract}
Aleksandar D. Rakic, Karl Bertling, Yah Leng Lim, Stephen J. Wilson, Milan Nikolic, Thomas Taimre, Dragan Indjin, Alexander Valavanis, Edmund H. Linfield, A. Giles Davies, Graeme Walker, Blake Ferguson, Tarl. W. Prow, Helmut Schaider, and H. Peter Soyer
\end{abstract}

A novel imaging and tissue analysis scheme in the terahertz frequency band has the benefits of simplicity, coherence, and high sensitivity.

Terahertz radiation has an inherently low penetration depth in hydrated biological tissue, so skin is an ideal target for imaging at terahertz frequencies. Examining structures within the surface layers of skin is a mainstay of diagnosis in pathology, and using several different frequency regions to image these can provide complementary information about skin structure and function. The research community has successfully developed diagnostic methods based on the response of tissue in the terahertz spectral region. ${ }^{1}$ However, to date, the absence of a compact, robust, inexpensive sensing solution has impeded general adoption of terahertz radiation for biological applications.

The quantum cascade laser (QCL) is one of the most promising radiation sources for imaging at terahertz frequencies. As with other lasers, QCLs exhibit self-mixing (SM), whereby re-injection of emitted radiation into the laser cavity affects the laser operating parameters. We can exploit this phenomenon for sensing purposes, using it to measure displacement, velocity, and fluid flow, as well as for coherent and incoherent imaging.

Here we present an imaging and tissue analysis scheme in the terahertz band that exploits the interferometric nature of coherent optical feedback in a terahertz QCL. SM interferometry is essentially a homodyne detection scheme, where the stable local oscillator signal (the QCL emission) is combined with the timedelayed version of itself. Figure 1 shows the basic structure and operating principles of our SM interferometer. The re-injected light interferes ('mixes') with the intra-cavity electric field, causing small variations in the fundamental laser parameters,

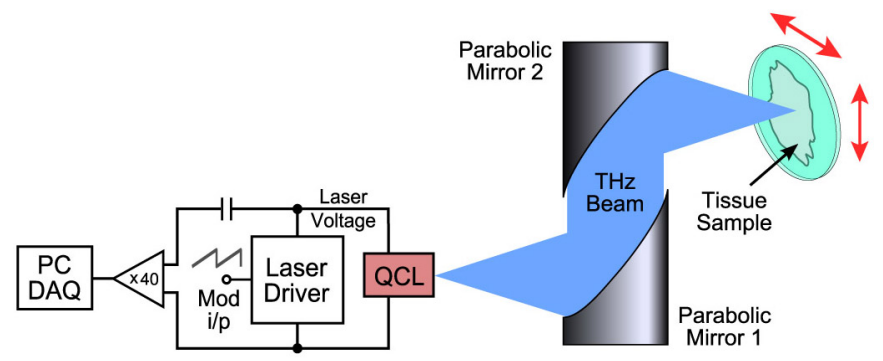

Figure 1. Schematic diagram of the setup for tissue imaging experiments. PC DAQ: Data acquisition. Mod i/p: Modulation input. QCL: Quantum cascade laser.

including the threshold gain, emitted power, lasing spectrum, and laser terminal voltage. ${ }^{2}$ While optical feedback affects almost all laser parameters, the two we can most conveniently monitor are the emitted optical power and the voltage across the laser terminals. Of these, monitoring the laser terminal voltage is preferable as it obviates the need for an external terahertz detector. ${ }^{3}$ The small voltage variation (referred to as the SM signal) depends on both the amplitude and phase of the electric field of the reflected laser beam. This results in a highly sensitive and compact sensing technique that can probe information about the complex reflectivity or refractive index of the external target.

We can create SM signals by exploiting temporal variations in optical length of the external cavity (through variation of its refractive index or the physical length), complex reflectivity of the target, or the laser frequency. The imaging process involves illuminating static objects with terahertz radiation. In other words, the objects being imaged are not changing during the signal acquisition. Therefore, we require some type of modulation to generate an SM signal. Here we opt for slow frequency modulation, ${ }^{4}$ which results in an SM waveform imprinted with 
the complex refractive index of the target. The sensitivity of this scheme is discussed in detail elsewhere. ${ }^{5}$

We performed tissue imaging experiments using porcine tissue and murine skin. We first acquired a 2D array of timedomain waveforms and then reduced each waveform to a corresponding array of numbers, forming an image. Figure 2 shows results of the reflection-mode imaging of a $3 \mathrm{~mm}-$ diameter skin biopsy from the transgenic laboratory mouse strain Cdk4::Tyr-NRAS.6,7 The sample in Figure 2(a) shows a barely visible stage 1 melanoma lesion on the left hand side of the sample. Figure 2(b) shows a signal strength reduction of the terahertz waveforms, while Figure 2(c) shows a corresponding phase reduction from the array of SM waveforms. We can relate the tumor plaque in this sample to features observable in the pair of terahertz image reductions.

In summary, we developed a highly sensitive and compact scheme that shows promise for biological imaging in the terahertz frequency range. Our approach offers the possibility of early detection of skin changes before they become apparent in visible images. Our results also point toward techniques for the characterization of healthy tissue types for the study of normal physiology and possible therapeutic approaches. Additionally, imaging of skin malignancy in animal models at terahertz

(a)

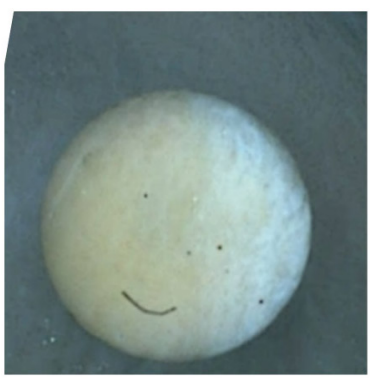

(b)

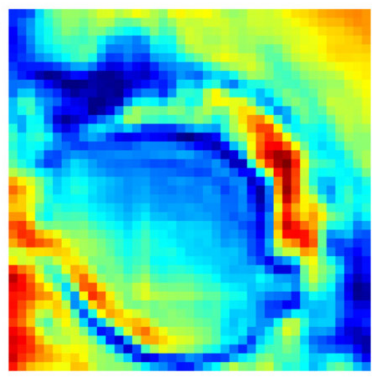

(c)

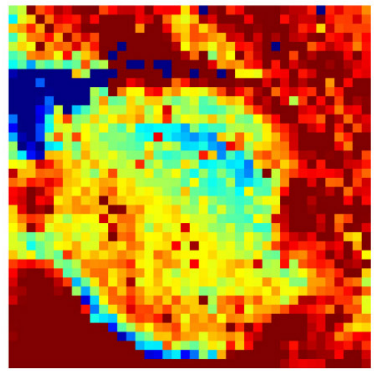

Figure 2. (a) Photograph of the $3 \mathrm{~mm}$ diameter skin biopsy from the Cdk4::Tyr-NRAS sample. (b) Trimmed total variation, showing a signal strength reduction of the terahertz self-mixing waveforms. (c) Trimmed last peak position, showing a phase-like reduction of the terahertz self-mixing waveforms. wavelengths shows earlier and potentially more powerful discrimination than is currently possible in the visible and IR regions of the spectrum.

Continuous improvements in terahertz QCL technology will lead to devices operating at higher temperatures and consequently more robust and compact self-mixing instruments for tissue characterization. In future work, we will investigate the origin of contrast in biological tissues based on water content and distribution, and we will assess the innate molecular response at terahertz frequencies. A clearer understanding of these factors will guide us towards discriminating a variety of skin pathologies.

This research was supported under the Australian Research Council's Discovery Projects funding scheme (DP 120 103703) and a Cancer Research UK Leeds Centre Development Fund Equipment award (Grant number C37059/A16369). We also acknowledge support of the ERC 'NOTES' and 'TOSCA' programs, the Royal Society, the Wolfson Foundation, and the European Cooperation in Science and Technology (COST) Action BM1205. Author Yah Leng Lim acknowledges support under the Queensland Government's Smart Futures Fellowships program.

\section{Author Information}

Aleksandar D. Rakic, Karl Bertling, Yah Leng Lim, Stephen J. Wilson, Milan Nikolic, and Thomas Taimre School of Information Technology and Electrical Engineering The University of Queensland

Brisbane, Australia

Dragan Indjin, Alexander Valavanis, Edmund H. Linfield, and A. Giles Davies

University of Leeds

Leeds, UK

Graeme Walker and Blake Ferguson

Queensland Institute of Medical Research

Herston, Australia

Tarl W. Prow, Helmut Schaider, and H. Peter Soyer

Translational Research Institute

Brisbane, Australia

\section{References}

1. C. Yu, S. Fan, Y. Sun, and E. Pickwell-MacPherson, The potential of terahertz imaging for cancer diagnosis: A review of investigations to date, Quant. Imag. Med. Surg. 2 (1), pp. 33-45, 2012. 
2. G. Giuliani, M. Norgia, S. Donati, and T. Bosch, Laser diode self-mixing technique for sensing applications, J. Opt. A, Pure Appl. Opt. 4 (6), pp. S283-S294, 2002.

3. P. Dean, Y. L. Lim, A. Valavanis, R. Kliese, M. Nikolić, P. Suraj Khanna, M. Lachab, et al., Terahertz imaging through self-mixing in a quantum cascade laser, Opt. Lett. 36 (13), pp. 2587-2589, 2011.

4. A. D. Rakić, D. Aleksandar, T. Taimre, K. Bertling, Y. L. Lim, P. Dean, D. Indjin, et al., Swept-frequency feedback interferometry using terahertz frequency QCLs: a method for imaging and materials analysis, Opt. Express 21 (19), pp. 22194-22205, 2013.

5. T. Taimre, K. Bertling, Y. L. Lim, P. Dean, D. Indjin, and A. D. Rakić, Methodology for materials analysis using swept-frequency feedback interferometry with terahertz frequency quantum cascade lasers, Opt. Express 22 (15), pp. 18633-18647, 2014.

6. E. M. Wurm, L.L. Lin, B. Ferguson, D. Lambie, T. W. Prow, G. J. Walker, and H. P. Soyer, A blueprint for staging of murine melanocytic lesions based on the $C d k 4(R 24 C / R 24 C):: T y r-N R A S(Q)(61 K)$ model, Exp. Dermatol. 21 (9), pp. 676-681, 2012.

7. E. Chai, B. Ferguson, T. Prow, P. Soyer, and G. Walker, Three-dimensional modelling for estimation of nevus count and probability of nevus-melanoma progression in a murine model, Pigm. Cell Mel. Res. 27 (2), pp. 317-319, 2014. 